\title{
HACIA UN CORRALÓN SOLIDARIO FAU
}

Eje 2: Tecnología para la construcción sustentable

Cremaschi, María Elisa ${ }^{1}$

Luna, María Eugenia ${ }^{2}$

Lombardi, Nelly ${ }^{3}$

\author{
${ }^{1}$ Laboratorio de Tecnología y Gestión Habitacional -LATEC-, FAU-UNLP, Argentina, elisacre@hotmail.com \\ ${ }^{2}$ Laboratorio de Tecnología y Gestión Habitacional -LATEC-, FAU-UNLP, Argentina, \\ corralonsolidario@fau.unlp.edu.ar \\ ${ }^{3}$ Laboratorio de Tecnología y Gestión Habitacional -LATEC-, FAU-UNLP, Argentina, \\ lombardinelly@gmail.com
}

\section{RESUMEN}

La Industria de la Construcción insume el $50 \%$ de los Recursos Naturales no Renovables y un gran consumo energético, lo cual la trasforma en una de las actividades menos sostenibles del planeta. Por otro lado, produce cuantiosos residuos ocasionados por demoliciones o rezagos de obra nueva que a menudo ocupan lugar en espacios públicos deteriorando el medio ambiente urbano. En consecuencia y en el marco de la educación universitaria actual, resulta imperioso que esta problemática sea atendida por la Universidad para generar estrategias que tiendan a optimizar el uso de los recursos, reducir y reutilizar todo lo posible, desarrollar técnicas constructivas convencionales o innovadoras, que sean eficientes y accesibles a la mayor cantidad de destinatarios posibles.

La Universidad Nacional de La Plata y específicamente la Facultad de Arquitectura y Urbanismo, exhibe una larga y fructífera actividad de extensión universitaria, honrando como pocas los postulados Reformistas y destinando recursos humanos y académicos al mejoramiento de las condiciones de vida de la sociedad.

Es por ello que en el marco del X CRETA resulta de interés compartir el proyecto de Extensión Universitaria "Hacia un Corralón Solidario FAU" que se desarrolla en el marco de la convocatoria Universidad, Cultura y Sociedad del Ministerio de Educación de la Nación. El proyecto propone vincular los materiales residuales de la construcción, rezagos de obras o en desuso, con la comunidad que los necesite y tendrá lugar en el nuevo espacio físico que la UNLP destinó a la FAU ubicado en el predio de los ex galpones de la Autoridad del Agua (Ada).

En esta oportunidad se pretende compartir los avances desarrollados hasta el momento para desarrollar una experiencia piloto con el objeto de recuperar, acopiar y distribuir materiales de construcción y destinarlos a actividades de extensión y a futuro, a nuevos destinos solidarios. En este sentido, consideramos que la actividad académica puede y debe involucrarse en la articulación 
de las políticas estatales para afrontar los desafíos de los años por venir y los avances que necesariamente deben desarrollarse en la industria de la construcción en comparación con los avances de otras industrias, tanto en cuestiones como el ahorro energético, el cuidado del medio ambiente, la integración social y el progreso económico de nuestra sociedad, que son las bases del desarrollo sustentable.

El equipo de trabajo con base en la FAU es interdisciplinario, trabajando docentes, graduados y estudiantes con orientaciones tecnológicas y destacada trayectoria en Proyectos de Extensión e Investigación.

\section{PALABRAS CLAVES: TECNOLOGÍA - TRANSFERENCIA - RCD - MATERIALES - COMUNIDAD}

\section{INTRODUCCIÓN}

El proyecto de Extensión Universitaria con trayectoria "Hacia un Corralón Solidario FAU" que se desarrolla en el marco de la convocatoria Universidad, Cultura y Sociedad del Ministerio de Educación de la Nación propone vincular los materiales residuales de la construcción, rezagos de obras o en desuso, con la comunidad que los necesite y tendrá lugar físico en el nuevo espacio que la UNLP destinó a la FAU ubicado en el predio de los galpones de la exAutoridad del Agua (Ada).

El equipo de trabajo con base en la FAU UNLP es interdisciplinario, trabajando docentes, graduados y estudiantes con orientaciones tecnológicas y destacada trayectoria en Proyectos de Extensión e Investigación. En la propuesta convergen dos Proyectos de Universidad, Cultura y Sociedad que son complementarios entre sí y una propuesta de Mayor Dedicación de Extensión (MDE): "Capacitación para el hábitat" dirigido por el Arq. Gustavo Páez ${ }^{1}$ (EU14-UNLP4022) y "Hacia un proyecto de vida" dirigido por el Arq. Gustavo Cremaschi ${ }^{2}$ (EU14-UNLP4583) y la MDE convocatoria 2016 "Capacitación para el hábitat, vivienda saludable y segura", presentado por la Arq. Nelly Lombardi .

Así mismo, el Laboratorio de Tecnología y Gestión Habitacional -LATEC-, dirigido por el ArqG. Cremaschi, desarrolla entre sus actividades el Programa de Vinculación Tecnológica -PVT- para profundizar la relación e interacción de la Facultad con los Sectores Productivo y Público, y colaborar con la obtención de nuevos desarrollos y tecnologías. Dentro de las tareas previstas del PVT se propone utilizar los denominados residuos de construcción y demolición ${ }^{4}$ (RCD) en base a trabajos de investigación realizados por el LATEC y en conjunto con el Instituto de Geomorfología y Suelos (IGS) de la Facultad de Ciencias Naturales y Museo.

Es por ello que el proyecto tiene como objetivo recuperar materiales de rezagos de obras que aún sean útiles, clasificarlos y depositarlos en el predio de la Universidad cedido a la FAU y destinarlos como insumos de actividades de investigación y extensión (Fig.1).

\footnotetext{
${ }^{1}$ Vicedecano y Profesor Titular de la FAU UNLP.

${ }^{2}$ Profesor Titular y Director del Laboratorio de Tecnología y Gestión Habitacional de FAU UNLP.

${ }^{3}$ Profesor Adjunto y Mayor Dedicación en Extensión de la FAU UNLP.

${ }^{4}$ Los residuos de construcción y demolición, son aquellos residuos que se generan como consecuencia de la demolición de edificaciones e infraestructuras y de la construcción de nuevas.
} 


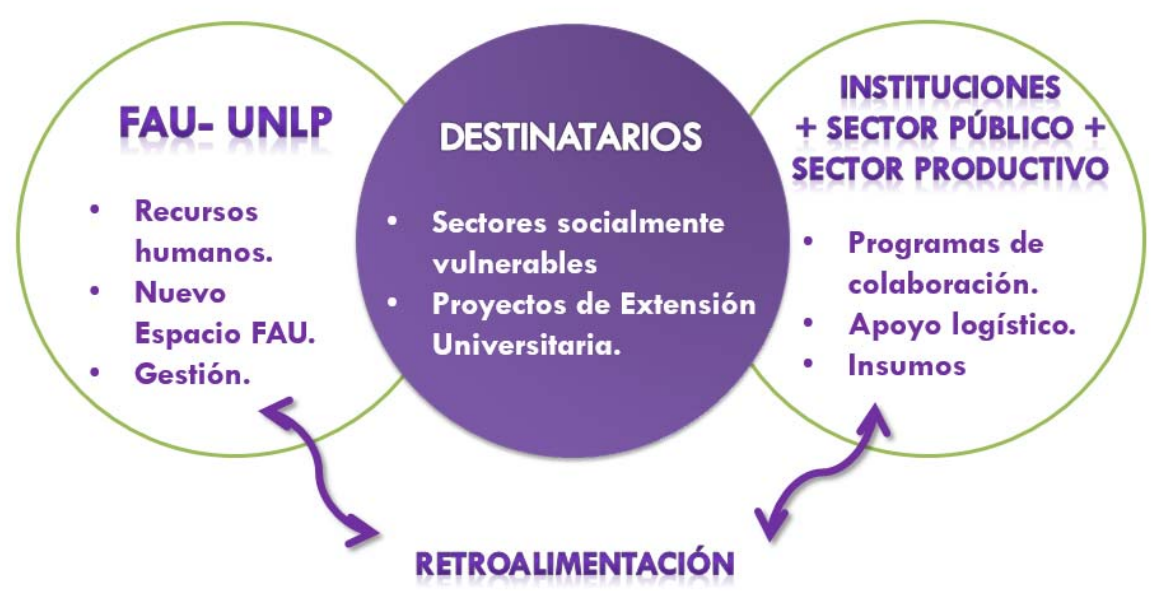

Fig.1. Actores intervinientes del Proyecto. Fuente: Elaboración propia. 2017.

\section{DESARROLLO}

\subsection{LA ACTIVIDAD DE LA CONSTRUCCIÓN}

La actividad de la construcción en el contexto de nuestra ciudad produce cuantiosos residuos ocasionados por demoliciones o rezagos de obra nueva que a menudo ocupan lugar en espacios públicos deteriorando el medio ambiente urbano (Fig.2). Por otro lado es conocido el impacto ambiental que produce la construcción y la Huella ecológica que generan todos los materiales desde su extracción, elaboración, transporte y puesta en obra, y las demoliciones posteriores a la vida útil de la misma.

Para comprender la dimensión de la problemática en cuestión, se debe tener en cuenta que la Industria de la Construcción consume el $50 \%$ de los Recursos Naturales no Renovables, lo cual la trasforma en una de las actividades menos sostenibles del planeta. Además, la explotación de los recursos naturales no es el único impacto ambiental que genera, es también una actividad con un gran consumo energético, problemática que debe ser atendida por la actividad académica.

En este marco, es importante destacar que los arquitectos pertenecientes a la comunidad educativa de la FAU comparten la docencia con el ejercicio liberal de la profesión, y en su mayoría ejecutan proyectos en los emprendimientos locales, encontrándose en contacto directo o indirecto con la ejecución de obras, corralones comerciales, fábricas de materiales, empresas constructoras, entre otros.

En este sentido, el proyecto apunta a dar destino a los residuos generados por la construcción y la demolición, enfocándose en las posibilidades de reutilización y reciclado que tienen los mismos, y su consecuente reducción. Lo que generaría, en consecuencia, una minimización del Impacto Ambiental y la aplicación del capital humano de la Universidad al mejoramiento de las condiciones de vida de la sociedad.

Si bien en una primera etapa se destinaran a proyectos de extensión, a futuro se proyecta ampliar los beneficiarios de estas acciones a la comunidad que los necesite permitiendo resolver problemas reales de la misma, como mejorar la precariedad de sus viviendas y las condiciones de habitabilidad. 
Se reducirían así, la producción de residuos urbanos y se fomentaría la reutilización de materiales que aún ofrecen prestaciones aprovechables en el mejoramiento del hábitat.
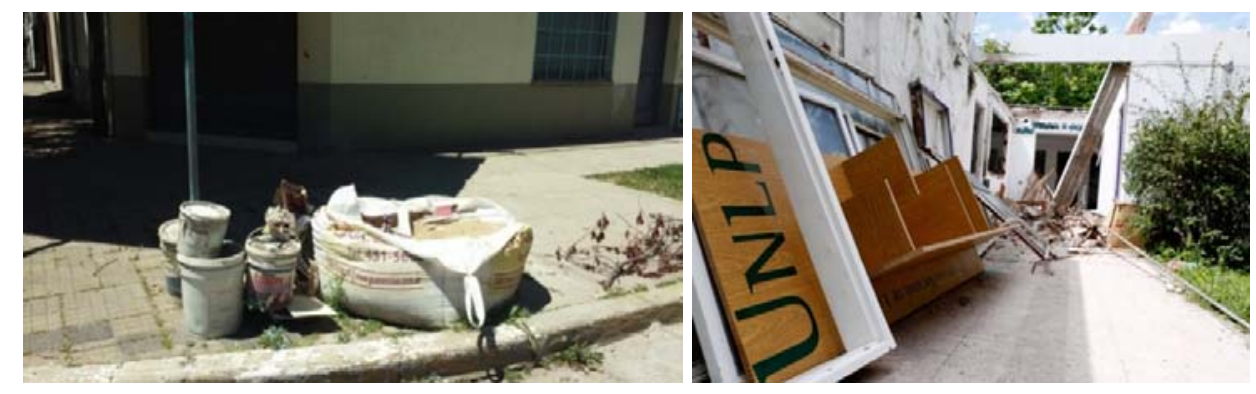

Fig.2. Residuos de construcción y demolición en la Ciudad de La Plata. Fuente: Elaboración propia. 2017/2018.

\subsection{EL PROGRAMA DE VINCULACIÓN TECNOLÓGICA Y SU NUEVO ESPACIO}

En el marco de las acciones del LATEC, el desarrollo del PVT y la obtención de un nuevo espacio físico, en el que las Cátedras, los Talleres, los extensionistas y las Unidades de Investigación de la Facultad, tengan un área en el que se puedan ensayar nuevas tecnologías, probar propuestas de investigación aplicada y contribuir al progreso técnico pasible de ser utilizado por el medio a partir de la generación de patentes, permitirá generar un círculo virtuoso entre la Universidad y la sociedad, así como el fortalecimiento de la relación de la Facultad con el sector productivo en general y el regional en particular.

El nuevo espacio, conseguido por la FAU a partir de largas tratativas, tiene lugar físico dentro de un predio actualmente perteneciente a la UNLP con varios galpones muy antiguos, que en el pasado fue propiedad de la Autoridad del Agua de la Provincia de Buenos Aires (Figura 3). La rehabilitación de este nuevo espacio, es imprescindible para desarrollar el proyecto y contribuir afrontar los desafíos de los años por venir y los avances que necesariamente deberán producirse en la industria de la construcción en comparación con los avances de otras industrias, en cuestiones como el ahorro energético, el cuidado del medio ambiente, la integración social y el progreso económico de nuestra sociedad, bases del desarrollo sustentable.

Uno de los principales objetivos que tiene el programa, es profundizar la Vinculación Tecnológica entre el sector científico- tecnológico universitario y los sectores productivo y empresarial, atendiendo también a las necesidades de orden municipal, provincial, nacional e internacional para mejorar la formación de las personas, la creación de conocimiento, la generación de desarrollos tecnológicos e innovaciones que demanden, tanto sectores productivos como organizaciones sociales. Si bien las actividades de vinculación se fueron consolidando en diferentes cátedras, laboratorios, centros e institutos de la Facultad, no se postulaba a la Vinculación Tecnológica como propósito institucional orientador de la creación de conocimientos y de la interacción con el medio capaz de incidir en las actividades académicas y científicas. 

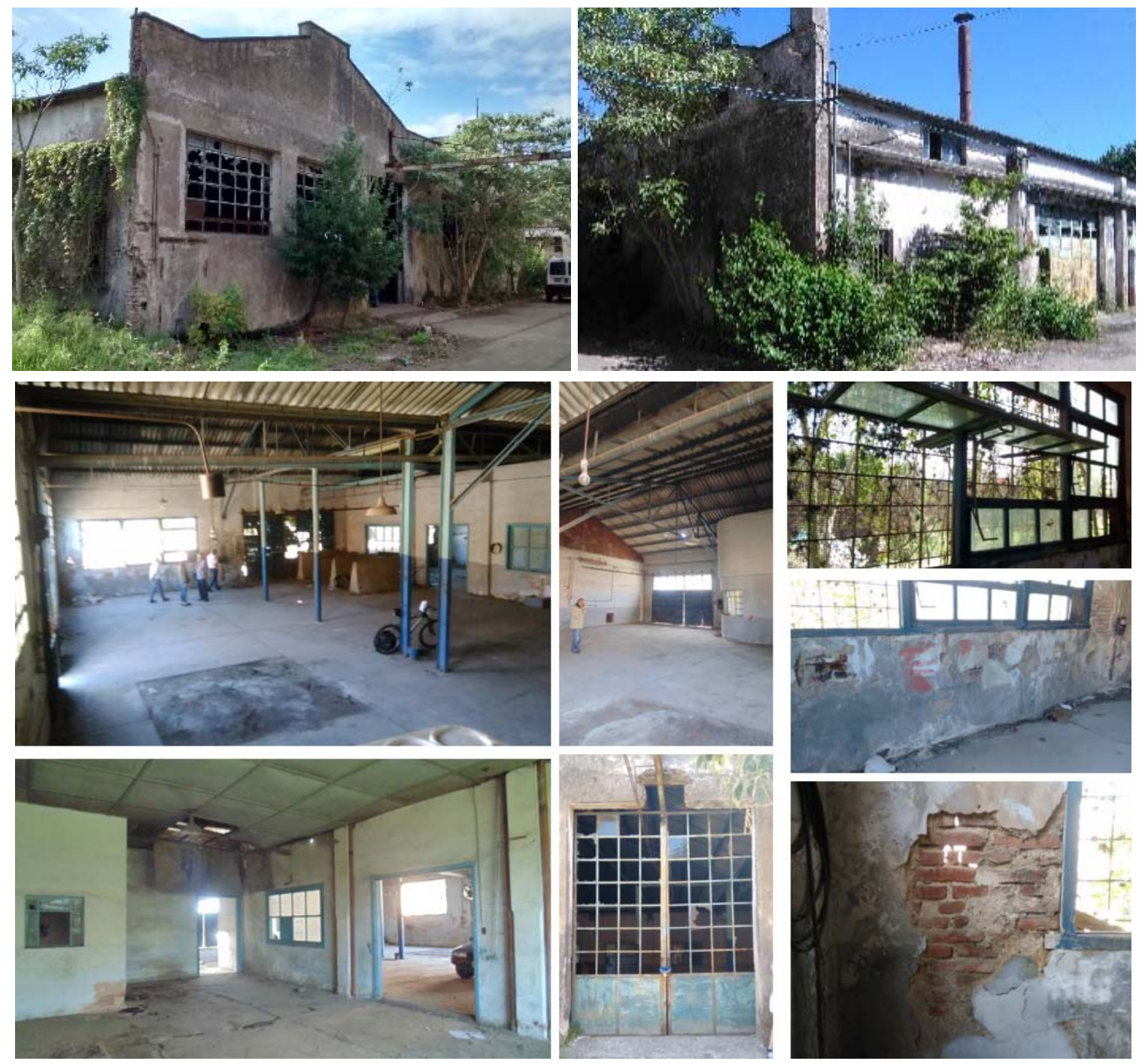

Fig.3. Nuevo espacio FAU -Ex ADA- en Predio Universitario. Fuente: Elaboración propia. 2016.

En este sentido es de orden prioritario que la FAU amplíe su rol y participación dentro de la sociedad, extendiendo su campo de acción, con una concepción abarcadora que genere nuevos espacios orientados, no solo al desarrollo productivo, sino también al intercambio de conocimiento y tecnología. Para ello se considera primordial conocer las necesidades y demandas de la sociedad, y a partir de allí, buscar respuestas generando las condiciones para que el conocimiento creado y desarrollado dentro de la Facultad tenga una inserción sinérgica con la comunidad. En este ámbito, se evidencia la necesidad de insumos de materiales en las actividades de extensión relacionadas con capacitaciones en el mejoramiento del hábitat de sectores vulnerables imposibilitados de afrontar los costos de la ejecución o mantenimiento de su vivienda.

En este sentido, la puesta en marcha del nuevo espacio de la FAU, impulsa la creación de actividades de transferencia de conocimientos para proyectar las capacidades institucionales hacia una innovación más inclusiva, siendo además un disparador para instaurar a la vinculación tecnológica como una actividad institucional. 


\subsection{LA PRUEBA PILOTO}

En una primera etapa del proyecto, se plantea la realización de una experiencia piloto con el objeto de recuperar, acopiar y distribuir materiales de construcción en actividades de extensión anteriormente mencionadas (Fig.4). Esta experiencia piloto, en escala acotada será dirigida a la actividad de extensión, e irá expandiendo progresivamente su actividad en función de las necesidades y del grado de acierto de la misma, hasta alcanzar el estado de ajuste y eficiencia necesarios para implementar la segunda etapa que incluye como destinatarios a la comunidad que necesite de este aporte, especialmente los sectores más vulnerables.

Para la organización y el desarrollo de esta etapa se tomaron como base experiencias ya realizadas en otras instituciones y para adaptarlas a las necesidades propias. Asimismo, se diseñó un protocolo preliminar de funcionamiento para la prueba piloto definido como un instrumento específico para regular todos aquellos aspectos que facilitan la continuidad del proyecto y su posterior puesta en marcha y replicabilidad.

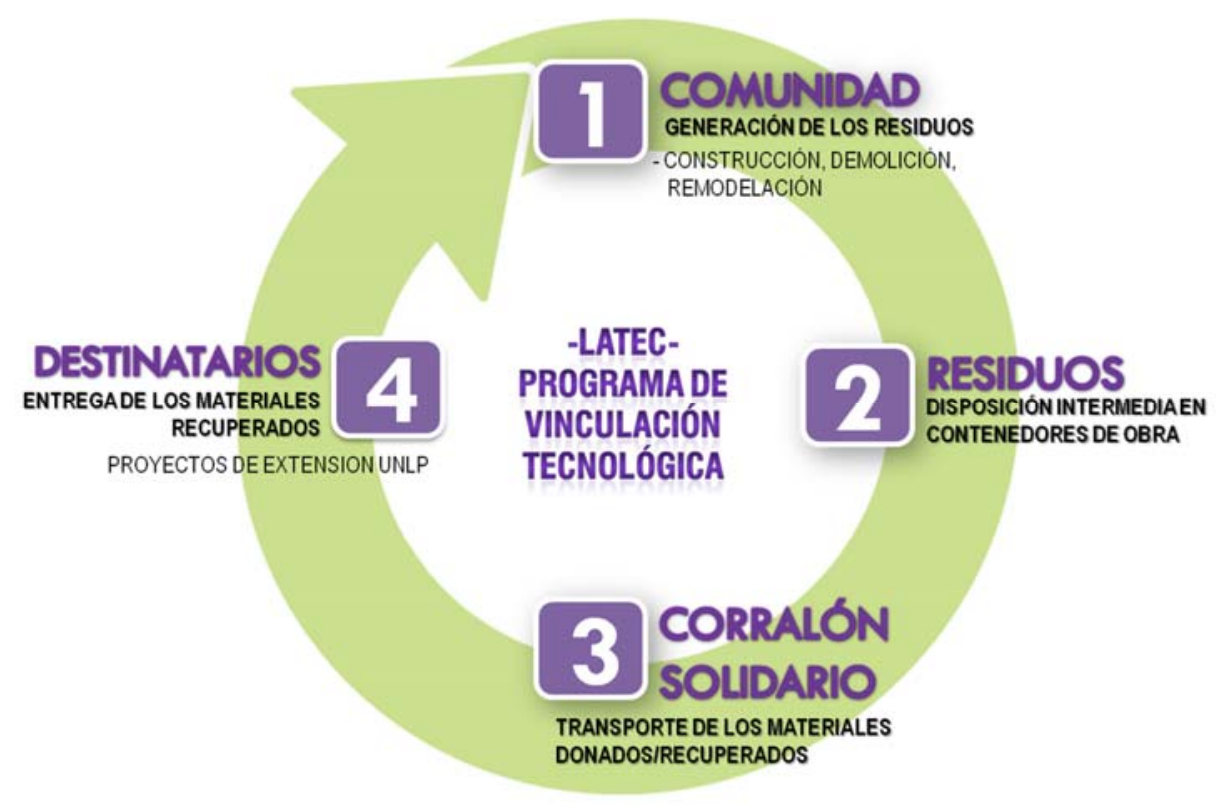

Fig.4. Esquema de funcionamiento del Proyecto. Fuente: Elaboración propia. 2018.

Como puede verse en la Figura 4, el circuito comienza con los materiales generados de rezagos de obras, demoliciones y/o sobrantes de comercialización y producción, que tengan la posibilidad de ser reutilizados en destinos comunitarios, de esta manera y a través del Corralón Solidario como articulador, se resuelven fundamentalmente dos problemas: por un lado el destino de residuos de obra y descarte de materiales y por otro, la carencia o necesidad detectada. Para que este circuito sea posible, el Corralón Solidario aceptará materiales residuales de obras de construcción y demolición que no sean peligrosos, tóxicos o nocivos para la salud. Para la prueba piloto los materiales a recolectar serán: arcillas, cal, cemento, plásticos, PVC, maderas, cartones, vidrios, acero, hierro, cobre, aluminio, estaño y zinc; residuos de tierra negra; concretos, cerámicos, ladrillos, 
arenas, bloques, baldosín y mortero, griferías, alacenas de cocina, bachas, inodoros, bidets, puertas y ventanas. No se aceptarán materiales humedecidos.

En esta primera etapa se propone comprometer tanto a la comunidad de la Facultad de Arquitectura y Urbanismo de la Universidad Nacional de La Plata, como a toda la sociedad en general. Teniendo en cuenta que una condición insoslayable de la aplicación de este proyecto es que sean destinos solidarios y acciones altruistas, se contempla una etapa de monitoreo y control de las donaciones, para llevar a cabo inspecciones para verificar el buen uso y destino de los materiales recuperados.

\subsection{PROTOCOLO DE FUNCIONAMIENTO}

Una de las premisas de este proyecto, es que sea replicable, por ello, se diseñó un protocolo de funcionamiento como un instrumento específico que regule todos aquellos aspectos que facilitan la continuidad del proyecto y su posterior puesta en marcha y replicabilidad.

El protocolo está compuesto por dos grandes guías: una guía de manejo residuos en la obra y una guía de gestión de materiales en el Corralón Solidario.

La primera está dirigida a toda persona, pública o privada que realice obras de construcción y demolición, con el objetivo de orientar en el manejo y disposición de los materiales sobrantes para disminuir el efecto sobre el ambiente y propender la ejecución de prácticas de reciclaje y re utilización de los materiales sobrantes. La segunda, tiene el objetivo de orientar el manejo y disposición de los materiales recuperados de obras, a partir de la llegada al Corralón Solidario, utilizando un software específico ya creado para registro y stock de materiales con planillas y formularios (Fig.5), disposición y almacenamiento de los materiales y destino final (donaciones) para que el manejo del corralón sea de forma ordenada y sistemática, asegurando su continuidad y posterior replicabilidad.

1. Guía de manejo de residuos en la obra

1.1. Clasificación de los residuos de construcción y demolición.

1.2. Disposición de los residuos.

1.3. Transporte de los mismos por parte del arquitecto solidario, entidad pública u otro medio.

2. Guía de gestión de materiales en el corralón solidario

2.1. Planillas

2.2. Formulario de donación de materiales.

2.3. Planilla de ingreso de materiales.

2.4. Clasificación de los materiales recibidos.

2.5. Disposición y acopio de los materiales. 


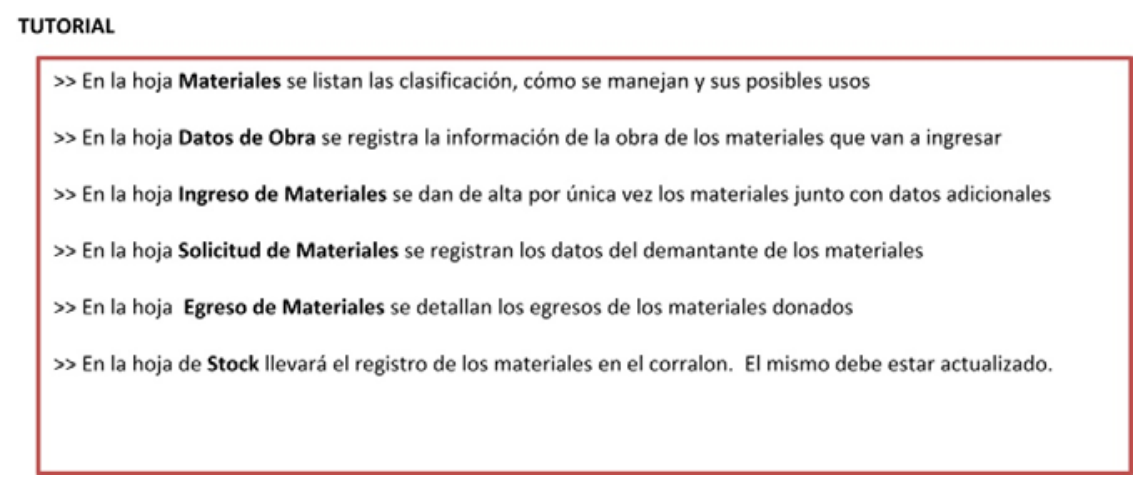

Fig.5. Tutorial de planillas para manejo de Corralón. Fuente: Elaboración propia. 2018.

\section{Destino de los materiales recuperados}

3.1. Planilla de solicitud de materiales. La misma deberá contener la información referente a los datos del solicitante, tales como: entidad o proyecto, responsable a cargo, localización, usos de los materiales a solicitar, entre otros.

\subsection{Evaluación de destinatarios y necesidades.}

\subsection{Planilla de egreso de materiales}

\subsection{Planilla de Stock de materiales actualizada}

4. Evaluación de resultados para la revisión y ajuste de la prueba piloto

4.1. Mapeo digital de donaciones y su posterior reutilización en sistemas de información geográfica 4.2. Monitoreo y control del buen uso de los materiales donados: una vez obtenido los materiales, llevar a cabo un control/inspección de los materiales y cómo fueron utilizados.

\subsection{Revisión y ajuste.}

\subsection{RESULTADOS OBTENIDOS}

El Proyecto ha despertado interés tanto en la comunidad en general como en la Universitaria. El primer objetivo cumplido, fue la aprobación del proyecto en la convocatoria "Universidad, Cultura y Sociedad" del Ministerio de Educación de la Nación, con el nombre de "Hacia un Corralón Solidario FAU", que ha sido el puntapié para dar los primeros pasos. A lo largo de estos meses, se han podido concretar vinculaciones con el Municipio de la Ciudad La Plata, específicamente con la Subsecretaria de Economía Solidaria (Director Francesco Vigliarolo) y la Dirección de Empleo (Directora Diana Prietto), así como también con empresas del mundo de la producción local(industria cerámica, sistemas en seco, madereras).Si bien el objetivo principal es poner en marcha el Corralón, estos primeros lazos, son imprescindibles para un buen desarrollo del proyecto.

Como se mencionó anteriormente, la rehabilitación del espacio físico que albergará el Corralón Solidario es vital para la puesta en marcha del proyecto. Es así como, siguiendo el proyecto de reacondicionamiento "Diagnóstico y propuesta para la rehabilitación de Edificio Universitario en el Predio Ex Ada" realizado en el marco las Prácticas Pre Profesionales Asistidas de la FAU- UNLP y coordinado por los Arqs. Gustavo Cremaschi/ María Elisa Cremaschi / Raúl Barandiarán (Marzo del 2017), por medio de la Dirección de Obras de la FAU-UNLP y de la Cooperativa "La Lucha por el trabajo" se realizaron las primeras tareas tales como: desmalezamiento y poda, limpieza general, 
reparación y colocación de vidrios de carpinterías exteriores, remodelación interior para oficina administrativa, entre otros.

Por otro lado, se avanzó en un vínculo con el medio productivo, que se encuentra en contacto directo o indirecto con la ejecución de obras, corralones comerciales, fábricas de materiales, empresas constructoras, entre otros. Hasta la fecha las empresas Ctibor, MP Maderera, Baukraft yTremex SRL mostraron interés en acompañar y colaborar con el proyecto.

Resulta de interés destacar el lazo con el Programa de compromiso ambiental de la UNLP "Recuperamos", dicho programa lleva adelante diferentes acciones que buscan generar un cambio en los hábitos de consumo y el manejo de los residuos, a través de minimizar, separar y recuperar los residuos sólidos urbanos que se generan en la comunidad universitaria, de esta manera se adquirirá soporte institucional en la temática medioambiental. Además el proyecto cuenta con un logo y material de difusión diseñados en el ámbito de la FAU.

Este Proyecto, propone ser la génesis de otro más amplio que requerirá de personal técnicoadministrativo dedicado exclusivamente a su desarrollo y puesta en marcha, y de personal especializado para la selección de los materiales. A efectos de lograr el lanzamiento oficial del Corralón Solidario se requiere que las entidades, transportadores, beneficiarios, comunidad de arquitectos, sitios de disposición temporal y final de escombros vayan en la misma línea y que esto permita que en la ciudad se dé un manejo apropiado de los RCD que repercuta en un menor impacto ambiental a la ciudad. Es importante destacar que, embarcarse en dicho proyecto requiere de un modelo de simulación previo para desarrollar el diseño preliminar de la propuesta.

\section{CONCLUSIONES}

Teniendo en cuenta las necesidades no satisfechas de amplios sectores de la sociedad en relación al acceso a un hábitat digno, la Universidad en general y la facultad de Arquitectura en particular no pueden omitir su participación proponiendo estrategias que tiendan a optimizar el uso de los recursos, en pos de dar respuesta a esta problemática, y a la vez contribuir con su acción al cuidado de medioambiente, altamente comprometido por una actividad directamente vinculada a su quehacer, como es la construcción de ciudad. Pero estos propósitos deben desarrollarse de forma creativa, vinculando sectores, optimizando procesos, convocando a las instituciones privadas y estatales en sus distintos niveles, de manera de crear conciencia y garantizar su perdurabilidad en el tiempo. El proyecto propone un cambio de paradigmas centrado en la sinergia, entre rezago y reutilización, deshecho y aprovechamiento, necesidad y solidaridad. También propone una facultad permeable, vinculada con el medio, sus necesidades y sus potencialidades, con un diálogo fructífero, bidireccional, capaz de interpretar su rol social y dar respuestas para mejorar la calidad de vida de la comunidad (Figura 6).
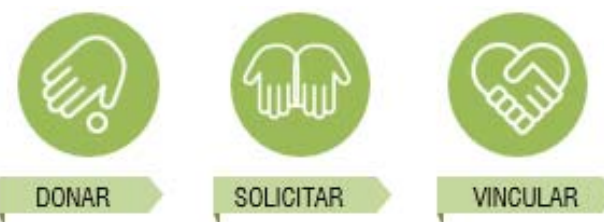

\section{corralonsolidario@fau.unlp.edu.ar}

Fig.6. Misiones. Fuente: Elaboración propia. 2018. 


\section{BIBLIOGRAFÍA}

Commoner, B. (1992) En paz con el planeta. Barcelona. Ed. Crítica.

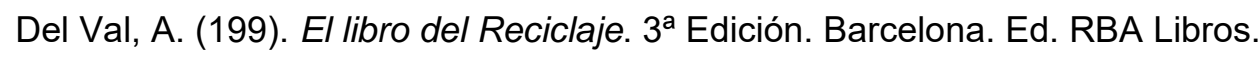

Edwards, B. (2009) Guía Básica de la Sostenibilidad. Segunda edición revisada y ampliada.

Hernandez Pezzi, C. (2007). Un Vitrubio ecológico. Principio y práctica del proyecto arquitectónico sostenible. Barcelona. Ed.G. Gilli.

Lynch, K.; Southworth, M. (2005) Echar a Perder: un análisis del deterioro.

Mercante, I. T. (2007). Caracterización de residuos de la construcción. Aplicación de los índices de generación a la gestión ambiental. Revista Cientifica de Primavera UCES, 24.

Varón, L., Sierra, D., \& Bedoya, L. (2011). Indural: un aporte significativo a la producción más limpia y la construcción sostenible. En Producción + limpia. 